\title{
Measurement strategies for Quantification of Carbon in Steel using EPMA
}

\author{
Philippe T. Pinard ${ }^{1}$, Anke Aretz ${ }^{1}$, Jürgen Börder ${ }^{2}$, John Donovan $^{3}$ and Silvia Richter ${ }^{1}$ \\ ${ }^{1}$ Central Facility for Electron Microscopy, RWTH Aachen University, Aachen, Germany. \\ 2 JEOL Germany GmbH, Eching, Germany \\ ${ }^{3}$ CAMCOR, University of Oregon, Eugene, Oregon
}

The quantification of low carbon concentration $(<1 \mathrm{wt} . \%)$ requires special procedures to minimize the influence of contamination. For steel, the calibration curve [1] or fixed background correction [2] methods are typically used with the assumption that the contamination rate on the unknown is equal to the one on the standard(s). One important requirement is, however, that the distance between measured positions should be large enough to prevent the contamination ring produced by one measurement to influence the next one. To measure sub-micrometer carbon-rich structures, this condition cannot be respected as the step size is much smaller than the radius of the contamination ring.

For well-separated points, only the cracked hydrocarbons inside the contamination ring influence the measured carbon intensity. From Monte Carlo simulations [3], 90\% of the carbon K-LIII x rays are emitted within a $340-\mathrm{nm}$ radius at $15 \mathrm{kV}$, well inside the $2-\mu \mathrm{m}$ contamination ring produced at this beam energy (Figure 1). When the distance between points is reduced, the contamination has a larger influence on the carbon intensity due to the overlap of the contamination rings. Figure 2 shows a beam line scan performed over a pure iron sample with a step size of $40 \mathrm{~nm}$ and a dwell time of $5 \mathrm{~s}$ at $15 \mathrm{kV}$ and $100 \mathrm{nA}$ on a JEOL JXA8530F. An oxygen jet was used during the acquisition. In the first micrometer, the behaviour is similar to the one for well-separated points as the electron beam stays within the contamination ring produced by the first point of the line scan. As the beam moves over the overlapped contamination rings of the previous measured points, the carbon intensity rapidly increases. A possible explanation for the following decrease is the depletion of hydrocarbon molecules. Only molecules located ahead of the line scan direction can be cracked as those downstream have already been. This example shows that not only the contamination rate influences the measurement of carbon but also dynamic effects related to the spacing between measurement positions.

To further study the influence of the step size, the precision of the carbon quantification was studied. The quantification was performed using a fixed background correction measured on pure iron and a $\mathrm{Fe}_{3} \mathrm{C}$ carbon standard. Only the points from the last $8 \mu \mathrm{m}$ were used for the quantification of the line scan. The measurements were performed under the same aforementioned acquisition conditions. Imprecision of EPMA measurements is the combination of random and systematic uncertainties [4]. The counting time was kept constant $(5 \mathrm{~s})$ to obtain similar random uncertainties. The systematic uncertainty was obtained by subtracting the random variance calculated based on Poisson statistics from the variance of the measured points. Figure 3 shows a better precision for well-separated points, but also some variations between the different reference samples. This illustrates the instability and inhomogeneity of the contamination level, even if all samples were polished and cleaned together. Overall, the precision of carbon quantification in low-alloyed steels is better than $0.05 \mathrm{wt} . \% \mathrm{C}$.

To better understand the contamination dynamics in order to improve the precision, a simulation model was created based on the same concepts used to study the structures produced by electron beam induced deposition [5]. The movement of hydrocarbon molecules is tracked through adsorption, desorption and diffusion as well as molecule-electron beam interactions. The influence of beam energy, electron dose, step size and anti-contamination devices (cold finger, plasma cleaner, O2 jet) can be studied using this model. A preliminary example of a simulated contamination ring is shown in Fig. 4. 
[1] ISO 16592 (2006)

[2] J.-F. Almagro et al, "Electron-probe Microanalysis of Light Elements; Measurements Methods and Certified Reference Materials", NPL, Teddington, United Kingdom (2002).

[3] D. Drouin et al, Scanning 29 (2007), pp. 92-101.

[4] R. B. Marinenko and S. Leigh, IOP Conf. Ser.: Mater. Sci. Eng. 7 (2010), p. 012017.

[5] D. A. Smith et al, Nanotechnology 18 (2008), p. 265308.

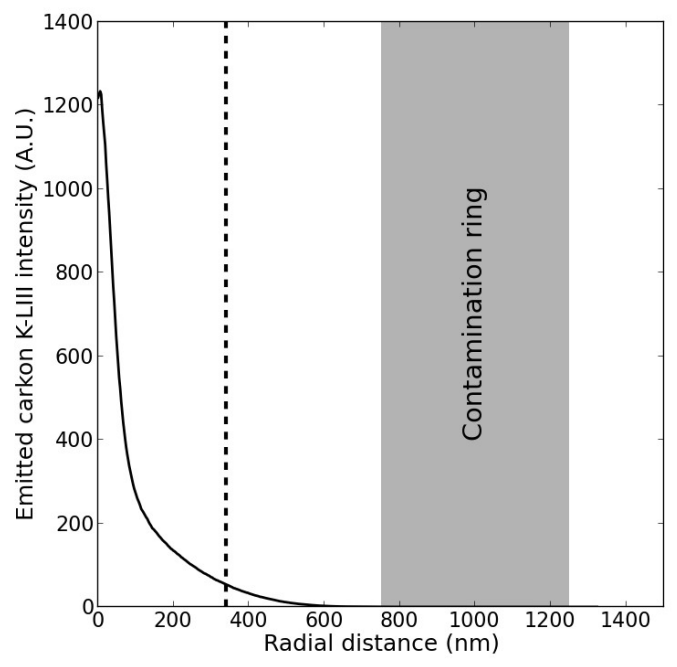

Fig. 1: Radial distribution of the emitted carbon K-LIII x-ray intensity for a $\mathrm{Fe}-1 \mathrm{wt} . \% \mathrm{C}$ sample $(15 \mathrm{kV}$ and $100-\mathrm{nm}$ beam diameter, Wincasino [3]). The dash line indicates the distance where $95 \%$ of the $\mathrm{x}$ rays have been emitted.

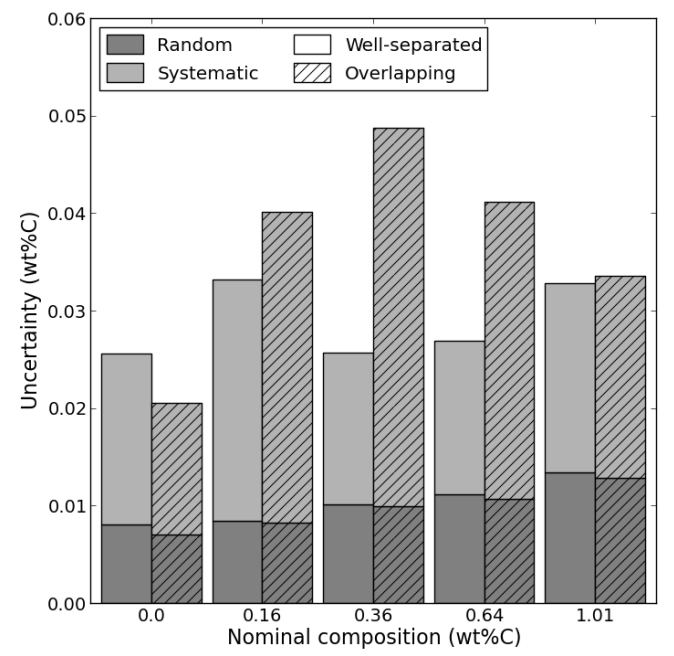

Fig. 3: Random and systematic uncertainties for well-separated and overlapping points acquisitions on reference samples.

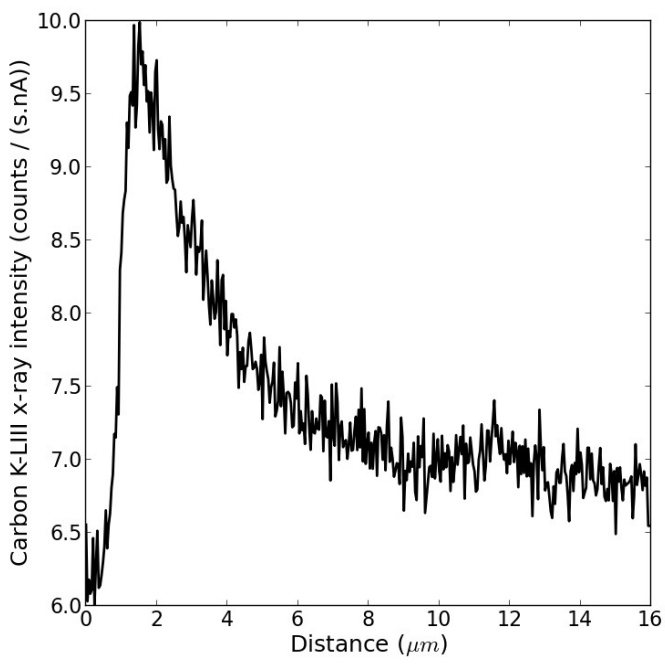

Fig. 2: Carbon K-LIII x-ray intensity profile along a beam line scan performed on a pure iron sample $(15 \mathrm{kV}, 100 \mathrm{nA}, 40 \mathrm{~nm}$ step size, $5 \mathrm{~s}$ dwell time, LDE2H crystal).

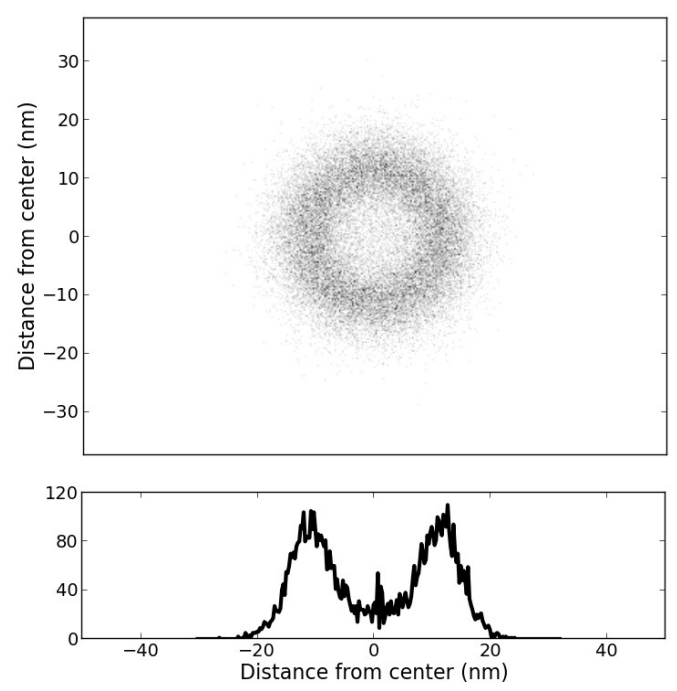

Fig. 4: Simulated contamination ring for a $10 \mathrm{~nm}$ beam diameter and extracted radial profile. 